\title{
When Defects Dominate: Rheology of Nanofibrillated Cellulose Suspensions
}

\author{
Martin A. Hubbe \\ Conventional rheological tests can be difficult to carry out in the case of \\ suspensions of nanofibrillated cellulose (NFC). Such suspensions tend to \\ migrate away from the walls of a rheometer device, leaving a low-viscosity \\ layer. The very high aspect ratio of typical nanofibrillated cellulose \\ particles favors formation of tangled clusters. But application of \\ hydrodynamic shear can cause fragmentation of those clusters. It is \\ proposed in this essay that some focus be placed on the fragments of \\ entangled clusters of NFC and interactions between them at their fractured \\ surfaces. The condition of near-uniform, defect-free structures of \\ nanocellulose spanning the volume within a sheared suspension might be \\ regarded as an unlikely circumstance. Isaac Newton started with a very \\ simple equation to start to understand rheology. It is proposed that a \\ similarly bold and simplified approach may be needed to account for the \\ effects of broken entangled clusters of NFC on flow phenomena, their \\ assessment, and their consequences related to industrial processes.
}

Keywords: Nanocellulose suspensions; Wall depletion; Shear thinning; Entanglement; Viscosity

Contact information: North Carolina State University; Dept. of Forest Biomaterials, Campus Box 8005, Raleigh, NC 27695-8005, USA; Email: hubbe@ncsu.edu

\section{Expectations}

Suppose that you woke up tomorrow morning in a completely transformed world. For instance, just imagine yourself as Alice in Wonderland, Rip van Winkle, or Jonathan Swift's protagonist Gulliver when visiting the land of the Lilliputians. A question that you would face, when confronted with such changed circumstances, is whether the "rules of the game" that you have relied upon for so long would still apply. On every previous morning when you woke up, you bent yourself at the hips and you were able to sit up. But when one is being held down by innumerable tiny threads, as in the case of Gulliver, such ingrained habits and routines just might not work.

Starting way back with Isaac Newton, the rules of the road for evaluating and predicting the flow of liquids and fluid suspensions have become well established. Newton's Philosophiae Naturalis Principia Mathematica, first published in 1687, set forth the well-known relationship between the applied shear stress and the induced flow of a simple, i.e. "Newtonian" liquid. Of course, real fluids may contain particles, soluble polymers, and either attractive or repulsive forces among them. Such features can give rise to flow phenomena that violate Newton's linear relationship between shear rate and shear stress. A few of these phenomena are encountered routinely by papermakers when they prepare and spread aqueous coatings of minerals onto the paper surface. For example, formulators of "coating colors" for paper know that such mixtures of pigments, binders, and additives in water tend to be "pseudo-plastic" when being changed from the stationary state, but that they can become "dilatant" when subjected to very high rates of shear. In addition, the same suspensions also can be "thixotropic," meaning that they show hysteresis. Their resistance to flow is different depending on whether one is increasing or 
decreasing the shear rate. With each added complexity, the science of rheology has expanded and become more enriched. In effect, more terms have been added to Newton's basic equation. In parallel with the development of concepts and mathematical expressions, new generations of precise equipment have emerged, making it possible to measure stresses and strains by rotational or oscillatory approaches. But then, along came nanofibrillated cellulose.

\section{Challenges}

I first became aware of the potentially troublesome nature of nanocellulose suspensions while working as a product development scientist for a large paper company. I was working to develop a piece of equipment to sense the zeta potential of fiber suspensions and to automatically run a titration to determine their cationic demand (Hubbe 2001). Someone told me that the best way to convince them that the device was ready for serious consideration would be to put a suspension of papermaking fibers in a tank, pump them continuously through a "flow loop", and set up my device to make automatic measurements repeatedly over a period of several days. That's what we did, and the first day everything was fine. On the second day it was necessary to open up the device and clean off a screen. On the third day the screen clogged up immediately, even when freshly cleaned. The continuous stirring and pumping of the ordinary kraft pulp suspension had converted the material into highly fibrillated cellulose - maybe even nanocellulose - and it no longer followed the rules of behavior for an ordinary pulp suspension.

To be fair, problems related to the rheology of suspensions of cellulosic fibers already were well known even before nanocellulose had come to the attention of papermakers. For example, Bennington et al. (1990) found that they needed to develop specialized equipment to be able to evaluate in the shear stress of fiber suspensions. If they employed ordinary "couette flow-type" equipment to make the measurements, the fibers tended to move away from the solid walls of the device, leaving a low-viscosity layer where "wall slip" would occur. To overcome this phenomenon, they attached rectangular baffles on both the inner and the outer walls of their couette device.

Issues of wall-slip and depletion of particles near to the wall surfaces of rheologymeasuring devices become yet more pronounced when using such equipment to evaluate nanocellulose suspensions (Hubbe et al. 2017). It was proposed in the cited work that nanocellulose particles undergo repeated entanglement followed by fragmentation of clusters of nanocellulose. The tendency for nanocellulose entities to become entangled with each other appears to exacerbate the tendency for solids-free zones to form near to the surfaces of the rheology-measuring equipment. When comparing data from many different studies of suspensions of nanofibrillated cellulose, there appeared to be two distinct tracks. One of the tracks, associated with relatively high values of shear stress at a given shear rate, appeared to be associated with contiguous structures filling the volume within the measuring cell of the device. A second track, with shear stress values as much as three factors of ten lower for the same solids content, appeared to represent systems in which the nanocellulose structures did not fill all of the volume. For the latter systems it was proposed that fragments of nanocellulose clusters existed apart from each other, with channels of relatively particle-free water between them. Koponen (2020) analyzed those findings and came to the conclusion that phenomena resembling wall slip are not isolated to wall regions when NFC suspensions are subjected to shear. Rather, such phenomena appear to occur at points throughout the volume of such suspensions. Evidence of 
relatively solids-free zones within sheared NFC suspensions were shown clearly by optical coherence tomography tests.

\section{Opportunities}

The aspect ratios of nanofibrillated cellulose are so high that they are seldom reported. When looking at micrographs to quantify NFC fibril widths, which are often in the range of 10 to $40 \mathrm{~nm}$, it is difficult to simultaneously try to figure out whether there is a continuous structure having a length of $10 \mu \mathrm{m}, 100 \mu \mathrm{m}$, or maybe even $1000 \mu \mathrm{m}$. The two types of dimension are hard to capture in the same micrograph. In addition, when NFC is produced mainly by mechanical shearing, one typically obtains a network-like structure rather than a suspension of individual fibrils.

It is proposed here that part of the answer is to stop expecting such material to follow the rules that rheologists have established for particle suspensions. What can be done instead? Here are some ideas:

- Treat the fragments of semi-broken NFC flocs as the suspended matter, rather than focusing on the individual NFC.

- Consider the potential contribution to resistance to flow when two such fragments of entangled NFC rub up against each other or mutually roll past each other in shear flow.

- Consider the flows of water into and out from such fragments of NFC clusters as they change their shapes to accommodate the somewhat crowded conditions within a sheared system.

- Attempt to model some of the fragmentation and re-entanglement phenomena.

I have read that Isaac Newton got quite angry when he first encountered people who questioned his theories. I have to admit that I am even less brave than Isaac, since at this point all I have offered are qualitative descriptions, not even an equation that could be tested. The challenge may lie in coming up with suitable simplifying models that capture the essence of rheological effects to be expected from collections of fragments of NFC flocs in a sheared suspension. Just think, if a graduate student is able to tackle this problem, maybe yet another term can be added to Newton's expression.

\section{References Cited}

Bennington, C. P. J., Kerekes, R. J., and Grace, J. R. (1990). "The yield stress of fibre suspensions," Can. J. Chem. Eng. 68, 748-757. DOI: 10.1002/cjce.5450680503

Hubbe, M. A. (2001). "Method for determining electrokinetic properties of papermaking furnish," U.S. Patent No. 6,176,974, Jan. 23.

Hubbe, M. A., Tayeb, P., Joyce, M., Tyagi, P., Kehoe, M., Dimic-Misic, K., and Pal, L. (2017). "Rheology of nanocellulose-rich aqueous suspensions: A Review,"

BioResources 12(4), 9556-9661. DOI: 10.15376/biores.12.1.2143-2233

Koponen, A. I. (2020). "The effect of consistency on the shear rheology of aqueous suspensions of cellulose micro- and nanofibrils: A review," Cellulose 27, 1879-1897.

DOI: 10.1007/s10570-019-02908-w

Newton, I. (1687). Philosophiae Naturalis Principia Mathematica, London. 